Running Head: CORD TESTOSTERONE AND CEREBRAL LATERALITY

\title{
Perinatal Testosterone Exposure and Cerebral Lateralisation in Adult Males: Evidence for the Callosal Hypothesis
}

\section{Lauren P. Hollier ${ }^{1,2}$, Murray T. Maybery ${ }^{2}$, Jeffrey A. Keelan ${ }^{3}$, Martha Hickey ${ }^{4}$, and Andrew J.O. Whitehouse ${ }^{1}$}

\footnotetext{
${ }^{1}$ Telethon Institute for Child Health Research, University of Western Australia

${ }^{2}$ Neurocognitive Development Unit, School of Psychology, University of Western Australia

${ }^{3}$ School of Women's and Infant's Health, University of Western Australia

${ }^{4}$ Department of Obstetrics and Gynaecology, University of Melbourne and the Royal Women's Hospital, Victoria Australia
}

Correspondence to:

Lauren Hollier

School of Psychology

University of Western Australia

35 Stirling Highway, Crawley

Western Australia, 6009

Phone: + 61864881300

Fax : + 61864881006

Email: lauren.hollier@graduate.uwa.edu.au 


\begin{abstract}
Two competing theories address the influence of fetal testosterone on cerebral laterality: one proposing exposure to high fetal testosterone concentrations is related to atypical lateralisation (Geschwind-Galaburda hypothesis), the other that high fetal testosterone concentrations exaggerate typical lateralisation (callosal hypothesis). The current study examined the relationship between cord testosterone concentrations and cerebral laterality for language and spatial memory in adulthood. Male participants with high $(>0.15 \mathrm{nmol})$ and low $(<0.10 \mathrm{nmol})$ cord testosterone levels were invited to take part in the study $(\mathrm{n}=18$ in each group). Cerebral laterality was measured using functional Transcranial Doppler Ultrasonography, while participants completed word generation and visual short-term memory tasks. Typical left lateralisation of language was more common in the hightestosterone group than in the low-testosterone group, $\chi^{2}=4.50, \mathrm{df}=1, p=.034$. Spatial memory laterality was unrelated to cord testosterone level. Our findings indicate that fetal testosterone exposure is related to language laterality in a direction that supports the callosal hypothesis.
\end{abstract}

Keywords: fetal testosterone, cerebral laterality, fTCD, language, visuospatial memory, Raine study 


\section{Introduction}

Functional hemispheric differences for language and visuospatial processes have been widely replicated in neuropsychology. Typically, the most crucial areas involved in language production are found in the left hemisphere, while the right hemisphere is more specialised for visuospatial functions (Whitehouse and Bishop, 2009). However, substantial variability between people has been identified. One suggested mechanism that may underlie variations in hemispheric asymmetry is exposure to differing concentrations of prenatal testosterone (Geschwind and Galaburda, 1987; Witelson, 1991; Witelson and Nowakowski, 1991).

Two prominent theories address the influence of fetal testosterone exposure on cerebral lateralisation. Geschwind and Galaburda (1987) proposed exposure to higher levels of fetal testosterone during critical periods of prenatal neurodevelopment shifts development of structures subserving certain functions, such as language, predominantly to the right hemisphere. They further suggest that the influence of prenatal testosterone exposure on lefthemisphere growth retardation would be more apparent in men. A second theory, the callosal hypothesis put forward by Witelson and colleagues (Witelson, 1991; Witelson and Nowakowski, 1991), suggests that, in men, higher concentrations of fetal testosterone increases axonal pruning in the corpus callosum, which leads to a more pronounced typical lateralisation of functions.

Studies examining the two competing theories have revealed mixed findings. Accurate measurement of fetal testosterone exposure presents a methodological challenge, and studies have typically used proxy measures. A commonly used indicator of fetal testosterone exposure is the second-to-fourth digit (2D:4D) ratio, where low 2D:4D ratio is suggestive of high fetal testosterone exposure (Manning, 2002). Kallai et al. (2005) found that low 2D:4D ratio was associated with decreased right posterior hippocampus volume and larger right middle hippocampus volume in 40 healthy female adults. These findings suggest levels of prenatal testosterone are related to development of laterality in sub-cortical regions. Bourne and Gray (2009) found lower 2D:4D digit ratios were associated with stronger right hemisphere lateralisation for visuospatial attention in a sample of 77 women. Similarly, a recently conducted fMRI study found a significant negative correlation between digit ratio and extent of functional visuospatial laterality (Kalmady et al., 2013). These studies suggest that higher prenatal testosterone exposure is associated with increased right hemisphere activation for visuospatial function. However, there is some doubt as to whether 2D:4D ratio is a reliable proxy measure for prenatal testosterone exposure, since the ratio has not shown a 
consistent relationship to more direct measures of prenatal testosterone (Dean and Sharpe, 2013; Hickey et al., 2010; Knickmeyer et al., 2011).

A few published studies have utilised a more direct measure of prenatal testosterone exposure through sampling amniotic fluid during the second trimester. In two studies (Grimshaw et al., 1995; Lust et al., 2010), cerebral lateralisation of offspring was measured using a dichotic listening task, which involves simultaneous presentation of different auditory stimuli to both ears. It is argued that participants with a right ear advantage (i.e. selecting the stimulus presented to the right ear more often than the one presented to the left ear) have left hemisphere dominance for language, and vice versa for a left ear advantage (Kimura, 1961). Both studies found an association between higher levels of testosterone in amniotic fluid and increased left hemisphere dominance for language, providing support for the callosal hypothesis (Grimshaw, et al., 1995; Lust, et al., 2010). However, neuroimaging studies have provided contradictory evidence. In support of the Geschwind and Galaburda hypothesis, amniotic testosterone concentrations have been shown to correlate positively with increasing rightward asymmetry of the isthmus (Chura et al., 2010). Mercure et al. (2009) measured functional lateralisation for perception of words and faces using event related potentials (ERPs) in 26 typically developing school-aged boys. No relationship was observed between amniotic testosterone levels and indices of cerebral lateralisation. While sampling amniotic fluid is a more direct measure than the 2D:4D ratio of the prenatal hormone environment, a limitation of this approach is that amniocenteses are performed only in high risk pregnancies and therefore research samples are unlikely to be representative of broader populations.

Recently, a new approach to sampling fetal testosterone has emerged through measurements of umbilical cord serum, which allow for larger and more representative sampling of pregnancies. Higher concentrations of cord testosterone have been associated with reduced vocabulary in boys at 2 and 5 years of age (Farrant et al., 2013; Hollier et al., 2013), increased risk of language delay in early childhood (Whitehouse et al., 2012), and reduced spatial ability in 6 year old girls (Jacklin et al., 1988). Umbilical cord blood can be easily collected from uncomplicated pregnancies following delivery, and so sex steroid concentrations reflect levels in fetal circulation at late gestation (Keelan et al., 2012). Most studies report that cord testosterone concentrations are consistently higher in males compared to females (Herruzo et al., 1993; Keelan, et al., 2012; Maccoby et al., 1979; Troisi et al., 2003). The main limitation of this approach is that cord hormone concentrations may not reflect those earlier in gestation, particularly during critical periods of fetal neurodevelopment (Cohen-Bendahan et al., 2005). However, there is increasing recognition from experimental 
studies, that effects of prenatal hormones on the development of neural regions are not restricted to the first two trimesters, and that final trimester levels may also play a role in neurodevelopment (Roselli et al., 2011; Zambrano et al., 2014).

\subsection{Present Study}

The current study examined the relationship between fetal testosterone and cerebral laterality in males, utilising umbilical cord blood collected at birth and a functional neuroimaging measure in early adulthood. Participants were drawn from a large populationbased pregnancy-cohort study and subjects were selected based on having low- or highconcentrations of testosterone measured from cord blood. Cerebral laterality was measured using functional Transcranial Doppler (fTCD), which uses ultrasound to measure eventrelated changes in blood-flow velocity in the middle cerebral arteries. fTCD is based on the premise that increases in neural activity leads to greater glucose and oxygen consumption that must be replenished via enhanced blood flow to the area (Lohmann et al., 2006). This method gives high correlations with existing 'gold standard' measures of cerebral lateralisation, such as the Wada technique (Knecht et al., 1998) and fMRI (Deppe et al., 2000).

Based on the Geschwind and Galaburda hypothesis it is predicted that high testosterone levels would be associated with less left hemisphere activation for language production and increased right hemisphere activation for visuospatial memory. Conversely, if the findings support the callosal hypothesis we would expect high testosterone levels to be associated with increased left hemisphere activation for language production and increased right hemisphere activation for visuospatial memory.

\section{Method}

\subsection{Participants}

Volunteers from the Western Australian Pregnancy Cohort (Raine) Study participated in the current study. Between May 1989 and November 1991, 2900 pregnant women were recruited from King Edward Memorial Hospital or nearby private practices. Eight-hundred and fifty-nine children (430 male; 429 female) had umbilical cord serum collected at birth. Right-handed male participants were selectively recruited to take part in the study based on the cord testosterone concentrations. In order to create high and low cord testosterone groups, recruitment commenced at the extreme ends of the cord testosterone spectrum and worked 
towards the median. A total of 36 participants (18 high testosterone, 18 low testosterone) agreed to complete the psychometric and neuroimaging testing. Participants in the low testosterone group had cord testosterone levels less than $0.10 \mathrm{nmol} / \mathrm{L}$, while those in the high testosterone group had levels greater than $0.15 \mathrm{nmol} / \mathrm{L}$ (Table 1).

\subsection{Procedure}

Immediately after delivery, mixed umbilical arterial-venous cord blood was collected, allowed to clot and serum was frozen at $-80 \mathrm{C}$. Serum was stored without thawing until the initial investigation as described by Keelan et al. (2012). Studies of steroid stability during long term storage confirm that serum samples can be stored for at least four decades at $-80 \mathrm{C}$ without loss or appreciable deterioration of steroid hormones (Bolelli et al., 1995; Stroud et al., 2007). Cord serum samples were thawed, aliquotted and shipped from Perth to South Australia for liquid chromatography-tandem mass spectrometry analysis (CPR Pharma Services Pty Ltd, Thebarton, SA).

Psychometric and fTCD testing was conducted when the participants were between 21 and 23 years of age. Participants were tested individually at a laboratory at the University of Western Australia. While fitted with the fTCD, the participants completed two experimental computer tasks, each running for approximately 20 minutes. The order of task presentation was counterbalanced, so that roughly half of the participants in each group received the word generation task followed by the spatial memory task, while the remainder were administered the tasks in the reverse order. Following the fTCD recording participants completed the psychometric tests, which took approximately 30 minutes.

\subsection{Testosterone Analysis}

Ten randomly selected cord-blood samples confirmed the absence of detectable maternal contamination (Whitehouse, et al., 2012). Assay performance was determined to be unaffected by up to three freeze-thaw cycles or $24 \mathrm{~h}$ at room temperature. Steroid analysis was performed blind to sample identity or characteristics. Total testosterone was measured by liquid chromatography-tandem mass spectrometry after solvent extraction as described by Keelan et al. (2012). Limit of quantitation was $0.025 \mathrm{ng} / \mathrm{ml}(0.08 \mathrm{nmol} / \mathrm{L})$. Inter-batch imprecision was 6-11\% $(n=24)$; recovery from cord serum was 93-98\%. Sex hormone binding globulin was measured by ELISA using a commercial kit (IBL International, Hamburg, Germany). BioT, representing the fraction of total testosterone either free (unsequestered by SHBG) or bound to serum albumin, was calculated by summing the 
concentrations of free testosterone and albumin-bound testosterone (Keelan, et al., 2012). Albumin levels were adjusted using published reference values to account for the decrease in serum albumin concentrations with gestational age (Zlotkin and Casselman, 1987).

\subsection{Psychometric Tests}

The Test for Reception of Grammar (TROG; Bishop, 2003) assesses receptive comprehension skills. For each of 80 items an array of four coloured pictures is presented and the participant is required to pick the picture that corresponds to the test-sentence that is read aloud by the experimenter. Each item consists of three distracters that can be either grammatical or lexical in nature. Scores can be transformed to standardised scores based on a distribution with a mean of 100 and standard deviation of 15 .

Wechsler Abbreviated Scale of Intelligence (WASI; Wechsler, 1999). Two subtests of the WASI were used to provide a measure of non-verbal ability. Matrix Reasoning is modelled on Raven's Progressive Matrices and involves a series of increasingly difficult visual pattern completion and analogy problems. For each question participants are required to choose from a multi-choice array the item that best completes the pattern. Block Design requires participants to rearrange a specific number of blocks to replicate a dual-coloured design, shown to them in a printed two-dimensional format, in a specified time limit. Matrix Reasoning and Block Design scores can be standardized and used to calculate a performance intelligence quotient (PIQ) score for a population mean of 100 and standard deviation of 15.

\subsection{Apparatus}

A functional Transcranial Doppler ultrasonography device (fTCD; DWL Multidop T2: manufacturer, DWL Elektronische Systeme, Singen, Germany) was used to measure changes in blood-flow velocity through the right and left middle cerebral arteries (MCAs).

Lateralisation of specific cognitive functions can be determined by comparing the evenrelated changes in blood flow velocity through the two MCAs. Participants were fitted with a flexible headset that held 2-MHz transducer probes that were placed over the two temporal skull windows. The experimental tasks were presented on a Phillips desktop computer and controlled by Presentation software (Neurobehavioural Systems), which sent markers to the Multidop system to denote the start of each epoch.

\subsection{Experimental Tasks}

A word generation paradigm was used to measure hemispheric lateralisation for language (Bishop et al., 2009). Participants were briefly cued to attend to the computer screen 
and after $5 \mathrm{~s}$ a letter from the alphabet appeared printed centrally on the screen. Upon presentation of the letter, participants were asked to silently generate as many words that they could think of that began with that letter. After $15 \mathrm{~s}$ participants were instructed on screen, to say out loud all the words they thought of during the silent generation period. Participants completed a total of 23 trials, using all letters of the alphabet except Q, X and Z.

A second task developed by (Whitehouse et al., 2009) was used to assess participants' lateralisation for visuospatial memory. Each trial displayed 17 white and 9 red circles, each 5 $\mathrm{cm}$ in diameter, which were evenly distributed across a black background, but not aligned in rows or columns (and therefore not conducive to verbal encoding). The red circles were scattered randomly across the screen and participants were asked to memorise their location. Participants were cued to attend to the computer screen, where the circles remained for $5 \mathrm{~s}$ and then disappeared, leaving a black screen for a period of $10 \mathrm{~s}$. Following this a tone sounded, and the circle array appeared again. In half of the 20 trials, the locations of one red and one white circle were swapped. Participants were asked to indicate whether the red circles were the same or different as those that appeared in the initial screen by raising the index finger on their left or right hand, respectively. The same sequence of trials was used for all participants.

\subsection{Laterality Index}

The fTCD data were analysed offline using the dopOSCCI software (Badcock, Holt, et al., 2012). The blood flow envelope from each probe was down-sampled at a rate of $100 \mathrm{~Hz}$. Following (Whitehouse and Bishop, 2009), epochs were set to begin $12 \mathrm{~s}$ before the cueing tone in both tasks, and to end at $40 \mathrm{~s}$ for the word generation task and $35 \mathrm{~s}$ for the spatial memory task. Following an artefact rejection procedure, where epochs with unusually high or low levels of activity were removed from the data, the accepted time-locked epochs were averaged. A baseline value was calculated as the mean velocity in the $10 \mathrm{~s}$ pre-cueing interval.

A laterality index (LI) was calculated by first computing the difference in the change of blood flow velocity between the left and right hemispheres during the predefined periods of greatest activation. In line with Whitehouse and Bishop (2009) these periods were defined as the silent generation phase of the word generation task ( $8-18 \mathrm{~s}$ after the start of each trial) and the recognition phase of the spatial memory task (22 - $32 \mathrm{~s}$ after the start of each trail). The LI was calculated as the mean blood flow velocity difference in a $2 \mathrm{~s}$ window centred on the peak value during these periods of greatest activation (Whitehouse and Bishop, 2009). The LI indicates the direction of laterality, where a positive index indicates greater left than right- 
hemisphere activation and vice versa for a negative index. More extreme LIs indicate a greater degree of lateralisation.

\subsection{Statistical Analysis}

Independent samples t-tests were used to (a) examine whether the participants in the high and low testosterone groups were significantly different in their chronological age, psychometric scores and BioT concentrations, (b) to confirm that the number of epochs accepted on the word generation and spatial memory paradigms did not differ significantly for the two groups, and (c) to compare the word generation and visuospatial memory LIs for these two groups. To further examine the relationship between laterality and testosterone level, participants with a positive LI were classified as left lateralised and those with a negative LI as right lateralised. A chi-square analysis was used to test for a significant difference in the frequency of right or left lateralisation as a function of testosterone level.

\section{Results}

Table 1 presents data on the chronological age, BioT concentrations and psychometric scores of participants in the two groups. Independent samples t-tests showed no significant differences between the two groups for chronological age, WASI PIQ or TROG scores. As expected based on group selection criteria, there was a statistically significant difference in BioT concentrations (cohen's $d=3.21$ ).

Analysis then turned to the data obtained through fTCD. There were no significant differences between the high and low testosterone groups for the number of epochs accepted for the word generation (low testosterone: $\mathrm{M}=20.83, \mathrm{SD}=2.09$; high testosterone: $\mathrm{M}=$ $21.22, \mathrm{SD}=.88 ; t(34)=.73, p=.47$ ) or spatial memory (low testosterone: $\mathrm{M}=18.28, \mathrm{SD}$ $=.96$; high testosterone; $\mathrm{M}=17.50, \mathrm{SD}=1.65 ; t(34)=1.73, p=.09)$ tasks. There were no significant correlations between either the WASI PIQ or TROG variables and word generation LI (PIQ: $r=-.276, p=.104$; TROG: $r=-.165, p=.337$ ) or visuospatial memory LI (PIQ: $r=-.273, p=.107$; TROG: $p=-.199, p=.246$ ).

Figure 1 presents LIs for the word generation and visuospatial memory tasks. The two groups did not differ significantly in LI for either word generation (low testosterone: $\mathrm{M}=$ 1.87, $\mathrm{SD}=2.36$; high testosterone: $\mathrm{M}=2.48, \mathrm{SD}=1.21 ; t(34)=.99, p=.33$ ) or spatial memory (low testosterone: $\mathrm{M}=-3.27, \mathrm{SD}=2.42$; high testosterone; $\mathrm{M}=-3.72, \mathrm{SD}=2.41 ; t$ $(34)=.57, p=.57)$. Furthermore, when examining the continuous variables, there was still no 
significant relationship between bioavailable testosterone concentrations and word generation LI $(r=.186, p=.277)$ or visuospatial memory LI $(r=-.003, p=.985)$. However, a significant difference emerged when we investigated the data in terms of left (a positive LI) and right lateralisation (a negative LI). Chi-square analysis revealed that right hemisphere language lateralisation was more likely for individuals from the low testosterone group (11\%) than those from the high testosterone group $(0 \%): \chi^{2}=4.50, \mathrm{df}=1, p=.03$. There was no significant difference in the frequencies of left and right lateralisation for visuospatial memory as a function of testosterone level: $\chi^{2}=.36, \mathrm{df}=1, p=.55$.

\section{Discussion}

We found that a significantly higher proportion of participants with low umbilical testosterone levels exhibited atypical lateralisation for language than those with high umbilical testosterone. This finding is in line with the callosal hypothesis, which proposes that higher levels of fetal testosterone increase axonal pruning in the corpus callosum, resulting in more pronounced typical lateralisation (Witelson, 1991). The current finding is consistent with results reported by both Lust et al. (2010) and Grimshaw et al. (1995) who found that higher levels of amniotic fluid testosterone were associated with increased left hemisphere dominance for language. However, it is important to note that the relationship between umbilical testosterone levels and language lateralisation was only apparent when participants were examined as either left or right lateralised. No significant relationship was observed when examining language lateralisation as a continuous variable. It may be possible that in a typically developing population there is not a high enough incidence of atypically lateralised individuals to find a significant relationship when examining the spectrum of laterality. Indeed, there were only four individuals in our sample with atypical language lateralisation (11\%), although this is within the range reported by other studies (Bishop, 1990; Deppe, et al., 2000; Flöel et al., 2005; Flöel et al., 2001; Whitehouse and Bishop, 2009). Further, due to the relatively small sample size of the current study, the results should be interpreted with caution and require replication. Despite the sample size and the reduction in power through the use of a dichotomous variable, it is important to explore the possibility that these findings are a true reflection of the underlying biology and that increased levels of fetal testosterone lead to a more pronounced left-hemisphere lateralisation for language.

Only one other investigation has examined the relationship between fetal testosterone and language lateralisation using direct measures similar to those used in the current study. 
Mercure et al. (2009) found no relationship between amniotic fluid testosterone levels and an ERP index of language lateralisation in typically developing boys, which is in contrast with the current study. However, one major difference between Mercure et al. (2009) and the current study is that we selectively recruited participants based on their fetal testosterone level. It is possible that for typically developing individuals, differences in lateralisation are more apparent when examining the extreme ends of the fetal testosterone spectrum.

No statistically significant difference was found between the high and low umbilical testosterone groups for visuospatial memory lateralisation. To date relatively little research has examined the relationship between fetal testosterone exposure and visuospatial lateralisation. The few studies that have examined this relationship have used indirect measurements. For example, Bourne and Gray (2009) and Kalmady et al. (2013) both found that a low digit ratio was associated with increased right hemisphere activation for visuospatial functions. However, the digit ratio is an approximation of fetal testosterone levels and has not shown a consistent relationship to more direct measures of fetal testosterone, such as concentrations from amniotic fluid (Dean and Sharpe, 2013; Knickmeyer, et al., 2011). While we argue that the use of cord blood provides a more direct measurement of fetal testosterone concentrations, further research into the relationship between fetal testosterone exposure and visuospatial laterality is warranted before firm conclusions can be drawn.

We found no significant relationship between either of the cognitive measures (WASI PIQ or TROG) and the LIs for either word generation or visuospatial memory. This finding is consistent with previous literature that has found no relationship between performance on word generation and visuospatial tasks and LIs measured using fTCD (Badcock, Nye, et al., 2012; Dräger and Knecht, 2002; Flöel, et al., 2001; Groen et al., 2011). Unfortunately, for the current study we did not record performance for either task during the fTCD recording and so we could not directly examine the relationship between task performance and LI.

Nevertheless, the previous fTCD research and the non-significant correlations of LIs with the cognitive measures in the present study indicate that there may be no relationship between task performance and cerebral laterality.

Although the use of umbilical cord blood to approximate levels of fetal testosterone allows for the non-invasive collection of representative participant samples, the main criticism of this approach is that testosterone levels measured at birth may not be representative of levels earlier in gestation (Cohen-Bendahan, et al., 2005). However, there is emerging evidence that neural regions subserving language function undergo growth during 
the third prenatal trimester (Quarello et al., 2008). Hence cord blood samples, in providing assessment of testosterone levels during the third trimester, allow unique insight into the possible effects of testosterone on late development of neural language centres. In addition, the current study's findings are supported by the Lust et al. (2010) and Grimshaw et al. (1995) studies, both of which used amniotic fluid sampled earlier in gestation. Currently there is no gold standard approach to the accurate measurement of fetal testosterone exposure during the critical periods of fetal brain development. The measurement of circulating fetal testosterone would require an invasive procedure, which would confer significant risk to the pregnancy.

Both the current study and Mercure et al. (2009), the only two studies to use relatively direct measures of lateralisation and fetal testosterone, have examined typically developing males. As yet the relationships between lateralisation of language or visuospatial functions and fetal hormone levels among typically developing females are unclear. Research in this area has typically focused on females with congenital adrenal hyperplasia (CAH). CAH is a genetic condition that is characterized by impairment of the enzyme that leads to cortisol biosynthesis, which results in an in utero pattern of adrenal cortisol deficiency and excess testosterone production (Mueller et al., 2008), present as early as week five of gestation (Mathews et al., 2004). Female fetuses with CAH are exposed to significantly higher concentrations of testosterone than is the case for normal pregnancies (Merke and Bornstein, 2005) and therefore provide an opportunity to examine the postnatal effects of exposure to varying levels of fetal testosterone. Three studies using dichotic listening to index lateralisation found no significant difference in cerebral laterality between girls with $\mathrm{CAH}$ and control participants (Helleday et al., 1994; Kelso et al., 2000; Mathews, et al., 2004). It is possible that more direct measurement of cerebral asymmetries, such as with fMRI or fTCD, may show a significant difference between girls with $\mathrm{CAH}$ and typically developing girls. Further research is required to investigate the relationship between fetal testosterone exposure and cerebral laterality in females.

In conclusion, the current study found a link between low levels of testosterone in cord blood and atypical language lateralisation in typically developing males, which provides evidence in favour of the callosal hypothesis of cerebral asymmetry. No relationship was found between cord testosterone levels and visuospatial laterality, indicating that late gestational testosterone exposure may not be related to the development of spatial lateralisation. 


\section{Acknowledgements}

The authors would like to acknowledge the National Health and Medical Research Council (NHMRC) and the Telethon Institute for Child Health Research (TICHR) for their long term contribution to funding the Raine study over the last 20 years. The androgen analysis was funded by Australian Rotary Health. Core Management of the Raine study has been funded by the University of Western Australia (UWA), Curtin University, the UWA Faculty of Medicine, Dentistry and Health Sciences, the Raine Medical Research Foundation, TICHR, and the Women's and Infants Research Foundation. AJOW is funded by a Career Development Fellowship (\#1004065) from the National Health and Medical Research Council (NHMRC). MH is funded by a Practitioner Fellowship from the NHMRC (\#1058935). JAK is funded by the Women and Infants' Research Foundation. The authors are extremely grateful to the study participants and their families, as well as the whole Raine Study Team which includes the Cohort Manager, Data Manager and data collection researchers. 


\section{References}

Badcock, N. A., Holt, G., Holden, A., Bishop, D. V. M., 2012. Doposcci: A functional transcranial doppler ultrasonography summary suite for the assessment of cerebral lateralization of cognitive function. Journal of Neuroscience Methods, 204(2), 383388.

Badcock, N. A., Nye, A., Bishop, D. V., 2012. Using functional transcranial doppler ultrasonography to assess language lateralisation: Influence of task and difficulty level. Laterality: Asymmetries of Body, Brain and Cognition, 17(6), 694-710.

Bishop, D. V. M., 1990. Handedness and developmental disorder: Cambridge University Press.

Bishop, D. V. M., 2003. The test for reception of grammar, version 2 (trog-2). London: Psychological Corporation

Bishop, D. V. M., Watt, H., Papadatou-Pastou, M., 2009. An efficient and reliable method for measuring cerebral lateralization during speech with functional transcranial doppler ultrasound. Neuropsychologia, 47(2), 587-590.

Bolelli, G., Muti, P., Micheli, A., Sciajno, R., Franceschetti, F., Krogh, V., et al., 1995. Validity for epidemiological studies of long-term cryoconservation of steroid and protein hormones in serum and plasma. Cancer Epidemiology Biomarkers \& Prevention, 4(5), 509-513.

Bourne, V. J., Gray, D. L., 2009. Hormone exposure and functional lateralisation: Examining the contributions of prenatal and later life hormonal exposure. Psychoneuroendocrinology, 34(8), 1214-1221.

Chura, L. R., Lombardo, M. V., Ashwin, E., Auyeung, B., Chakrabarti, B., Bullmore, E. T., et al., 2010. Organizational effects of fetal testosterone on human corpus callosum size and asymmetry. Psychoneuroendocrinology, 35(1), 122-132.

Cohen-Bendahan, C. C. C., van de Beek, C., Berenbaum, S. A., 2005. Prenatal sex hormone effects on child and adult sex-typed behavior: Methods and findings. Neuroscience \& Biobehavioral Reviews, 29(2), 353-384.

Dean, A., Sharpe, R. M., 2013. Anogenital distance or digit length ratio as measures of fetal androgen exposure: Relationship to male reproductive development and its disorders. Journal of Clinical Endocrinology \& Metabolism, 98(6), 2230-2238. 
Deppe, M., Knecht, S., Papke, K., Lohmann, H., Fleischer, H., Heindel, W., et al., 2000. Assessment of hemispheric language lateralization[colon] a comparison between fmri and ftcd. Journal of Cerebral Blood Flow and Metabolism, 20(2), 263-268.

Dräger, B., Knecht, S., 2002. When finding words becomes difficult: Is there activation of the subdominant hemisphere? NeuroImage, 16(3), 794-800.

Farrant, B. M., Mattes, E., Keelan, J. A., Hickey, M., Whitehouse, A. J. O., 2013. Fetal testosterone, socio-emotional engagement and language development. Infant and Child Development, 22(2), 119-132.

Flöel, A., Buyx, A., Breitenstein, C., Lohmann, H., Knecht, S., 2005. Hemispheric lateralization of spatial attention in right- and left-hemispheric language dominance. Behavioural Brain Research, 158(2), 269-275.

Flöel, A., Knecht, S., Lohmann, H., Deppe, M., Sommer, J., Dräger, B., et al., 2001. Language and spatial attention can lateralize to the same hemisphere in healthy humans. Neurology, 57(6), 1018-1024.

Geschwind, N., Galaburda, A. M., 1987. Cerebral lateralisation: Biological mechamisms, associations and pathology. Cambridge, MA: MIT Press.

Grimshaw, G. M., Bryden, M. P., Finegan, J.-A. K., 1995. Relations between prenatal testosterone and cerebral lateralization in children. Neuropsychology, 9(1), 68-79.

Groen, M. A., Whitehouse, A. J. O., Badcock, N. A., Bishop, D. V. M., 2011. Where were those rabbits? A new paradigm to determine cerebral lateralisation of visuospatial memory function in children. Neuropsychologia, 49(12), 3265-3271.

Helleday, J., Siwers, B., Ritzén, E. M., Hugdahl, K., 1994. Normal lateralization for handedness and ear advantage in a verbal dichotic listening task in women with congenital adrenal hyperplasia (cah). Neuropsychologia, 32(7), 875-880.

Herruzo, A. J., Mozas, J., Alarcón, J. L., López, J. M., Molina, R., Molto, L., et al., 1993. Sex differences in serum hormone levels in umbilical vein blood. International Journal of Gynecology \& Obstetrics, 41(1), 37-41.

Hickey, M., Doherty, D. A., Hart, R., Norman, R. J., Mattes, E., Atkinson, H. C., et al., 2010. Maternal and umbilical cord androgen concentrations do not predict digit ratio $(2 \mathrm{~d}: 4 \mathrm{~d})$ in girls: A prospective cohort study. Psychoneuroendocrinology, 35(8), 1235-1244.

Hollier, L. P., Mattes, E., Maybery, M. T., Keelan, J. A., Hickey, M., Whitehouse, A. J. O., 2013. The association between perinatal testosterone concentration and early vocabulary development: A prospective cohort study. Biological Psychology, 92(2), 212-215. 
Jacklin, C. N., Wilcox, K. T., Maccoby, E. E., 1988. Neonatal sex-steroid hormones and cognitive abilities at six years. Developmental Psychobiology, 21(6), 567-574.

Kallai, J., Csathó, Á., Kövér, F., Makány, T., Nemes, J., Horváth, K., et al., 2005. Mriassessed volume of left and right hippocampi in females correlates with the relative length of the second and fourth fingers (the $2 \mathrm{~d}: 4 \mathrm{~d}$ ratio). Psychiatry Research: Neuroimaging, 140(2), 199-210.

Kalmady, S. V., Agarwal, S. M., Shivakumar, V., Jose, D., Venkatasubramanian, G., Reddy, Y. C. J., 2013. Revisiting geschwind's hypothesis on brain lateralisation: A functional mri study of digit ratio (2d:4d) and sex interaction effects on spatial working memory. Laterality: Asymmetries of Body, Brain and Cognition, 18(5), 625-640.

Keelan, J. A., Mattes, E., Tan, H., Dinan, A., Newnham, J. P., Whitehouse, A. J. O., et al., 2012. Androgen concentrations in umbilical cord blood and their association with maternal, fetal and obstetric factors. PLoS ONE, 7(8), e42827.

Kelso, W. M., Nicholls, M. E. R., Warne, G. L., Zacharin, M., 2000. Cerebral lateralization and cognitive functioning in patients with congenital adrenal hyperplasia. Neuropsychology, 14(3), 370-378.

Kimura, D., 1961. Cerebral-dominance and the perception of verbal stimuli. Canadian Journal of Psychology, 15(3), 166-171.

Knecht, S., Deppe, M., Ebner, A., Henningsen, H., Huber, T., Jokeit, H., et al., 1998. Noninvasive determination of language lateralization by functional transcranial doppler sonography : A comparison with the wada test. Stroke, 29(1), 82-86.

Knickmeyer, R. C., Woolson, S., Hamer, R. M., Konneker, T., Gilmore, J. H., 2011. 2d:4d ratios in the first 2 years of life: Stability and relation to testosterone exposure and sensitivity. Hormones and Behavior, 60(3), 256-263.

Lohmann, H., Ringelstein, E. B., Knecht, S., 2006. Functional transcranial doppler. In R. W. Baumgartner (Ed.), Handbook on neurovascular ultrasound (pp. 251-260). Basel: Karger.

Lust, J. M., Geuze, R. H., Van de Beek, C., Cohen-Kettenis, P. T., Groothuis, A. G. G., Bouma, A., 2010. Sex specific effect of prenatal testosterone on language lateralization in children. Neuropsychologia, 48(2), 536-540.

Maccoby, E. E., Doering, C. H., Jacklin, C. N., Kraemer, H., 1979. Concentrations of sex hormones in umbilical-cord blood: Their relation to sex and birth order of infants. Child Development, 50(3), 632-642. 
Manning, J. T., 2002. Digit ratio: A pointer to fertility, behavior, and health. New Jersey: Rutgers University Press.

Mathews, G. A., Fane, B. A., Pasterski, V. L., Conway, G. S., Brook, C., Hines, M., 2004. Androgenic influences on neural asymmetry: Handedness and language lateralization in individuals with congenital adrenal hyperplasia. Psychoneuroendocrinology, 29(6), 810-822.

Mercure, E., Ashwin, E., Dick, F., Halit, H., Auyeung, B., Baron-Cohen, S., et al., 2009. Iq, fetal testosterone and individual variability in children's functional lateralization. Neuropsychologia, 47(12), 2537-2543.

Merke, D. P., Bornstein, S. R., 2005. Congenital adrenal hyperplasia. The Lancet, 365(9477), 2125-2136.

Mueller, S. C., Temple, V., Oh, E., VanRyzin, C., Williams, A., Cornwell, B., et al., 2008. Early androgen exposure modulates spatial cognition in congenital adrenal hyperplasia (cah). Psychoneuroendocrinology, 33(7), 973-980.

Quarello, E., Stirnemann, J., Ville, Y., Guibaud, L., 2008. Assessment of fetal sylvian fissure operculization between 22 and 32 weeks: A subjective approach. Ultrasound in Obstetrics and Gynecology, 32(1), 44-49.

Roselli, C. E., Estill, C. T., Stadelman, H. L., Meaker, M., Stormshak, F., 2011. Separate critical periods exist for testosterone-induced differentiation of the brain and genitals in sheep. Endocrinology, 152(6), 2409-2415.

Stroud, L. R., Solomon, C., Shenassa, E., Papandonatos, G., Niaura, R., Lipsitt, L. P., et al., 2007. Long-term stability of maternal prenatal steroid hormones from the national collaborative perinatal project: Still valid after all these years. Psychoneuroendocrinology, 32(2), 140-150.

Troisi, R., Potischman, N., Roberts, J. M., Harger, G., Markovic, N., Cole, B., et al., 2003. Correlation of serum hormone concentrations in maternal and umbilical cord samples. Cancer Epidemiology Biomarkers \& Prevention, 12(5), 452-456.

Wechsler, D., 1999. Wechsler abbreviated scale of intelligence. San Antonio, TX: The Psychological Corporation and Harcourt Brace.

Whitehouse, A. J. O., Badcock, N., Groen, M. A., Bishop, D. V., 2009. Reliability of a novel paradigm for determining hemispheric lateralization of visuospatial function. Journal of the International Neuropsychological Society, 15(06), 1028-1032.

Whitehouse, A. J. O., Bishop, D. V. M., 2009. Hemispheric division of function is the result of independent probabilistic biases. Neuropsychologia, 47(8-9), 1938-1943. 
Whitehouse, A. J. O., Mattes, E., Maybery, M. T., Sawyer, M. G., Jacoby, P., Keelan, J. A., et al., 2012. Sex-specific associations between umbilical cord blood testosterone levels and language delay in early childhood. Journal of Child Psychology and Psychiatry, 53(7), 726-734.

Witelson, S. F., 1991. Neural sexual mosaicism: Sexual differentiation of the human temporo-parietal region for functional asymmetry. Psychoneuroendocrinology, 16(13), 131-153.

Witelson, S. F., Nowakowski, R. S., 1991. Left out axoms make men right: A hypothesis for the origin of handedness and functional asymmetry. Neuropsychologia, 29(4), 327333.

Zambrano, E., Guzmán, C., Rodríguez-González, G. L., Durand-Carbajal, M., Nathanielsz, P. W., 2014. Fetal programming of sexual development and reproductive function. Molecular and Cellular Endocrinology, 382(1), 538-549.

Zlotkin, S., Casselman, C., 1987. Percentile estimates of reference values for total protein and albumin in sera of premature infants (less than 37 weeks of gestation). Clin Chem, $33(3), 411-413$. 